PDFlib PLOP: PDF Linearization, Optimization, Protection

Page inserted by evaluation version www.pdflib.com - sales@pdflib.com 


\title{
Carotenoids and Antioxidant Nutrients following Burn Injury ${ }^{a}$
}

\author{
CHERYL L. ROCK,,$^{b}$ JORGE L. RODRIGUEZ, ${ }^{b}$ \\ RUBINA KHILNANI, ${ }^{b}$ DEBORAH A. LOWN, ${ }^{b}$ \\ AND ROBERT S. PARKER ${ }^{c}$ \\ ${ }^{b}$ Program in Human Nutrition and Department of Surgery \\ The University of Michigan \\ M5539 SPHII \\ Ann Arbor, Michigan 48109-2029 \\ and

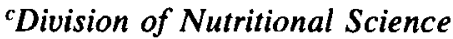 \\ Cornell University \\ Ithaca, New York 14853-0001
}

Carotenoids are among the circulating antioxidant micronutrients, and plasma levels of these compounds generally reflect dietary intake, when possible confounding factors such as body mass are considered. ${ }^{1.2}$ Patients who have suffered burn injury are abruptly withdrawn from food and are fed via enteral formula diet. Until recently, such diets provided essential nutrients (including vitamin $C$ and vitamin E) but have not provided carotenoids. Oxidant damage following burn injury can result in lipid peroxidation and may promote acute lung injury, and antioxidant micronutrients may play a protective role.

The purpose of this study is (1) to identify the dynamic pattern of plasma antioxidant nutrients following burn injury, using high performance liquid chromatography (HPLC) methods, and (2) to evaluate the effect of administering betacarotene enterally for 21 days following burn injury. Serial carotenoid levels in adipose tissue samples are also being quantified. Outcome variables include measures of lung function and biological indicators of immune function, including interleukin-8 levels in bronchial secretions.

\section{METHODS}

Subjects are patients with burn injury who are admitted to The University of Michigan Hospitals: 18-65 years of age, major burn injury ( $>20 \%$ total body surface area [BSA], full thickness [FT]), within two postburn days at recruitment, in a stable clinical state. Patients are fed a low carotenoid enteral formula diet (without additional micronutrient supplementation) within 24-48 hours of admission and treated with a standard protocol of fluid resuscitation, early excision of burned areas, and skin grafting. Blood samples are obtained at baseline and at least twice weekly during the 21-day (three-week) study period. Patients are randomly

${ }^{a}$ Work described in this poster paper was supported in part by a grant from Ross Laboratories. 
assigned to receive $30 \mathrm{mg} /$ day beta-carotene or placebo capsules (Hoffmann-La Roche, Nutley, NJ).

Plasma levels of vitamin E (alpha- and gamma-tocopherol), retinol, and the predominant carotenoids (beta-carotene, alpha-carotene, lycopene, beta-cryptoxanthin, and lutein) are quantified with HPLC methods. ${ }^{3,4}$ Plasma cholesterol and triglycerides are also measured in each blood sample for interpretation of fatsoluble nutrient levels. Plasma vitamin $C$ levels are measured with a derivative spectrophotometric method. ${ }^{5}$ Tissue analysis is achieved using the extraction method of Parker. ${ }^{6}$ Measures of immune status are obtained by collaborators in the Department of Surgery.

TABLE 1. Selected Plasma Micronutrient Levels (Mean $\pm \mathrm{SD}, \mathrm{n}=12)^{a}$

\begin{tabular}{cccl}
\hline Baseline & Week 1 & Week 2 & \multicolumn{1}{c}{ Week 3 } \\
\hline Beta-Carotene (umol/L) & & & \\
P:0.0547 \pm 0.0459 & $0.0832 \pm 0.0585$ & $0.0452 \pm 0.0356$ & $0.0283 \pm 0.0292$ \\
B:0.0451 \pm 0.0058 & $0.0892 \pm 0.1039$ & $0.0996 \pm 0.0368$ & 0.6196 \\
Lycopene (umol/L) & & & \\
P:0.2090 \pm 0.0931 & $0.2659 \pm 0.1324$ & $0.1295 \pm 0.0679$ & $0.0651 \pm 0.0546$ \\
B:0.1513 \pm 0.0663 & $0.1548 \pm 0.0969$ & $0.0935 \pm 0.0091$ & 0.2476 \\
Retinol (ug/dL) & & & \\
P:8.7595 \pm 4.3841 & $13.191 \pm 6.3953$ & $21.750 \pm 12.573$ & $20.588 \pm 7.9522$ \\
B:8.8546 \pm 5.5431 & $9.1792 \pm 0.2447$ & $17.554 \pm 15.056$ & 30.949 \\
Alpha-Tocopherol (mg/dL) & & & \\
P:0.4649 \pm 0.1871 & $0.7238 \pm 0.4608$ & $0.8670 \pm 0.4337$ & $0.7783 \pm 0.4571$ \\
B:0.4248 \pm 0.0042 & $0.5831 \pm 0.0024$ & $0.6928 \pm 0.2330$ & 0.9082 \\
Vitamin C (mg/dL) & & & \\
P:0.4430 \pm 0.1690 & $0.8426 \pm 0.5560$ & $1.2254 \pm 0.6832$ & $0.9876 \pm 0.3300$ \\
B:0.4098 \pm 0.3040 & $0.8294 \pm 0.0984$ & $0.7292 \pm 0.1292$ & 1.1268 \\
\hline
\end{tabular}

${ }^{a} \mathrm{P}$ indicates placebo group, and $\mathrm{B}$ indicates beta-carotene supplemented group.

\section{RESULTS AND CONCLUSIONS}

A total of 12 patients ( 4 females, 8 males) who met study criteria have been recruited, and 8 of these survived the 21-day study period. Descriptive data on these subjects are: $43 \pm 15$ years, $33.4 \pm 8.3$ weight $[\mathrm{kg}] /$ height $\left[\mathrm{m}^{2}\right]$ body mass index $, 47 \pm 18 \% \mathrm{BSA}, 41 \pm 21 \% \mathrm{FT}$. Descriptive data on selected plasma micronutrients are listed in TABLE 1.

Overall, plasma carotenoid levels were initially low and tended to remain low unless supplemented. Beta-carotene supplementation was not associated with increased plasma retinol levels; instead, increased retinol levels occurred in association with recovery. A time-dependent increase in certain chromatographic peaks was observed, among the more polar carotenoids on the chromatogram, following burn injury. These compounds may be oxidized carotenoid metabolites.

Plasma levels of tocopherols and vitamin $C$ were initially low, compared with normal values, but these levels increase during treatment in association with enteral diets. Correlations between plasma tocopherols and lipid levels were not 
observed in these patients. Plasma levels of alpha-tocopherol were inversely correlated with interleukin-8 levels $(r=-0.698)$ in bronchial secretions.

In conclusion, levels of circulating carotenoids remain low during low carotenoid enteral formula feeding unless supplemented. The dynamic pattern of plasma antioxidant nutrients following burn injury indicates reduced circulating levels and increased risk of medical problems secondary to oxidant stress. Supplementation may be necessary to promote maintenance of antioxidant defense mechanisms in these patients.

\section{ACKNOWLEDGMENTS}

The authors wish to express their gratitude to Dr. Hemmige Bhagavan of Hoffmann-La Roche, Inc. (Nutley, NJ) for kindly providing beta-carotene, placebo capsules and carotenoid standards.

\section{REFERENCES}

1. Micozzi, M. S., E. Brown, B. K. Edwards, J. G. Bieri, P. R. Taylor, F. Khachik, G. R. Beecher \& J. C. Smith. 1992. Am. J. Clin. Nutr. 55: 1120-1125.

2. Rock, C. L., M. E. Swendseid, R. A. Jacob \& R. W. MCKeE. 1992. J. Nutr. 122: $128-135$.

3. Bieri, J. G., E. D. Brown \& J. C. Smith. 1985. J. Liquid Chromatogr. 8: 473-484.

4. Bieri, J. G., T. J. Tolliver \& G. L. Catignani. 1979. Am. J. Clin. Nutr. 32: 2143-4149.

5. Omaye, S. T., J. D. Turnbull \& H. E. Sauberlich. 1979. Methods Enzymol. 62: 3-11.

6. Parker, R. S. 1988. Am. J. Clin. Nutr. 47: 33-36. 